\title{
Epidemiology and Prevalence of Type 2 Diabetes Mellitus in Children with Obesity
}

\author{
Vidit Kulshrestha and Syed M. Shahid
}

\section{ABSTRACT}

The epidemiology and prevalence of Type 2 Diabetes Mellitus (T2DM) in today's youth and tomorrow's adults, is currently in alarming stages and goes hand in hand with Childhood Obesity $(\mathrm{CO})$. This association causes detrimental impacts on child health and around $68 \%$ global population today are affected by it. T2DM is initiated in children with obesity as insulin functioning is resisted, which further leads to an inflammation and damage in the pancreas leading to adverse effects in later stages of life. Many scientific studies have been conducted to understand this link and does Body Mass Index (BMI) contribute to this epidemiology and complications related to T2DM and Obesity in children. Alarming effects, both physically and internally on the pancreas are observed because of these conditions and also causes fat deposition in the body organs like pancreas leading to increased Body Mass Index (BMI) and Waist Circumference (WC). This association can be caused due to genetic factor during infancy, but this has still not been validated as relevant researches are still underway. Important studies like SEARCH for Diabetes in Youth have contributed to giving important and useful findings about this association but still much needs to be achieved to make future progress in overcoming this burden on child health and ensure that Sustainable Developmental Goal (SDG) 3 is achieved at a global level.

Keywords: Body Mass Index (BMI), Childhood Obesity (CO), Insulin Resistance (IR), Type 2 Diabetes Mellitus (T2DM).

Submitted : January 28, 2021

Published : February 18, 2021

ISSN: 2593-8339

DOI: $10.24018 /$ ejmed.2021.3.1.693

\section{Vidit Kulshreshta}

School of Health Science,

Eastern Institute of Technology (EIT),

Auckland Campus, New Zealand.

(e-mail: kulshreshtavidit1@gmail.com)

Syed M. Shahid*

School of Health Science,

Eastern Institute of Technology (EIT),

Auckland Campus, New Zealand.

(e-mail: sshahid@eit.ac.nz)

*Corresponding Author

\section{INTRODUCTION}

Prevalence of Childhood Obesity (CO) is being reported in large number of studies across globe each day. About one-third of children globally, fall in the category of being overweight or obese. Obesity related risk factors have been considered as a metabolic syndrome and generally occur in lower- and middle- income countries of the world [1]. There are newly emerging evidences that show there exists a direct link between Type 2 Diabetes Mellitus (T2DM) and children living with Obesity and poor Body Mass Index (BMI). Most children and youth face problems of achieving the optimal levels of glucose and are also at risks of adult life health complications. Both Obesity and T2DM pose major healthcare challenges and dangerous effects on the economical, personal and societal factor [2].

As per findings, there are several factors that lead to T2DM, which can be accounted for the reason that during childhood, Obesity causes effects on body's capability to potentially use Insulin which leads to abnormal levels of glucose in the blood and hence lead to T2DM [3]. Approximately, $8.3 \%$ of the global population encounters T2DM, with around 191,000 cases in childhood for which Obesity is the causative factor. In America, around 17\% of children between 2-19 years of age fall in the category of
Obesity and whose BMI ranks above the 95th Percentile of the growth chart prepared by the Centers for Disease Control and Prevention (CDC) [4], [5].

This results in a stronger relationship shared by Obesity and Diabetes. Children with T2DM have poor levels of treatment and face severe complications like Insulin Resistance (IR), Hyperglycemia, Hypertension, Fat deposition and can also encounter Cardiovascular Diseases in future [4]. The premature onset of T2DM in children of the Obese category exposes them to metabolic abnormalities in body and long-life term macro and micro complications which might also lead to deaths. The pathophysiological phenomenon of pancreas and its $\beta$-Cells in obese children produce insulin but at a lower rate due to which blood glucose levels are abnormal in nature [5].

The purpose of this brief review report is to understand and recapitulate on the findings retrieved about the epidemiology and prevalence of obesity and T2DM and the biological relationship that is shared by both. This also provides recommendations for its disease management and prevention policies that can be worked upon and a proper roadmap may be prepared for fulfilling future healthcare requirements. 


\section{BiologicAl AsSOciation OF CHILDHOOD OBESITY AND TYPE 2 DiABETES MELlitus}

Over the past two decades T2DM prevalence and epidemiology has been increased to around ten times in countries like USA, UK, France, etc and the increase is most evident in age groups equivalent to Childhood Obesity in contrast to adults with T2DM, complications are seen much more in children who are obese [1]. IR and its inability to function can be accounted for the links that T2DM and CO hold. This resistance of the insulin hormone reduces the ability of muscles, fat, and liver cells to use the biological sugars in the body to convert it into metabolic energy.

Scientific studies show that adipose tissues of the body show most resistant to insulin and hence it can be said that obese people have problems related to glucose levels in the body due to poor glucose absorption and assimilation of glucose. Various factors are linked to IR like hereditary factor, ageing and ethnicity. Lifestyle factors like alcohol consumption, tobacco usage and stress contribute to T2DM. In American region, the cases of obesity in children have increased up to 3 times in the last few decades [6]. It is evident through studies that if children have BMI greater than 30, then there are higher chances of developing T2DM in your lifetime. Visceral body fat leads to T2DM related health problems since abdominal fat is the one whose cells release chemical agents that prove to be inflammatory that prove to be inflammatory and reduces the biological capacity of the biological capacity of the body to utilize insulin produce by it [6].

As per current findings, obese children are at risk of developing T2DM by the age of 25 [6]. It is presented in another study that majority of children ranked at high BMI and obese had four times risk of encountering T2DM by the age of 25 in respect to normal BMI children [7]. T2DM currently is being identified in children at an alarming rate due to factors like poor diet and lack of physical activity. As per a study conducted on children facing weight changes, it can be understood that overweight children had positive links to T2DM development. Hence, it is said that more obese the child is, the more are the chances of getting T2DM at an early age [7].

\section{Methodology}

A quick research of the National Center for Biotechnology (NCBI) and PubMed search engines was conducted and a few recently published peer reviewed research articles during last 5 years that were relevant to the topic were filtered out and selected for this review.

A study was published in 2019 using Mendelian Randomization method and conducted to identify the link shared between CO and T2DM [8]. This study further evaluated the complications that are posed by Obesity and risk factors in children who are overweight and how they encounter T2DM in later stages of life [8].

Another study was conducted to understand the trends of association between Obesity and events of T2DM in future by taking into consideration the BMI [9]. This was a cohort study which took into consideration the Clinical Practice Research Datalink (CPRD) from the UK. This data consisted of clinical diagnosis, prescriptions and test results. Around 369,362 individuals ranging from 2 to 15 years of age as of 2013 were sampled and their individual BMI were recorded.

A study selected for this review, conducted to understand the trajectories of BMI of children who are obese and whether they develop T2DM in future [10]. In this study 13,345 children born in Helsinki and Finland during the period of 1934-1944 were considered. The mean BMI was divided into cohorts at 11 years of age and their BMI and glucose tolerance were recorded.

\section{MAIN FINDINGS AND DisCUSSION FROM THE SELECTED STUDIES}

This section summarizes the key findings from each of the selected research articles and describes the significant observations relevant to the topic of this review report.

The study conducted on the Mendelian Randomization (MR) analysis has reported that CO leads to $16 \%$ higher chances of developing T2DM [8]. Similar results were observed using the statistical mean and median methods. It was found that per-unit rise in the log-odds of having obese children was linked with 0.192-Standard Deviation Rise in BMI as depicted in Table 1. The MR Analysis method was used because of its global usage in evaluation of links between T2DM and CO to understand its outcomes [8]. The results clearly linked to T2DM and has resulted in its epidemiological prevalence globally in recent years. The study also shows that MR Analyses reports that the $\mathrm{CO}$ leads to a decrease in High-Density Lipoprotein Cholesterol (HDL-c) amounts in body as shown in Table I. The analyses also reported that the Obesity was also linked to higher levels of BMI, Waist Circumference (WC), Hip Circumference (HC) and Waist and Hip Ratio (WHR). All observations of cohorts reported that children who were obese were bound to rise in adult T2DM levels [8]. It was also observed that rise in BMI of children was linked to $83 \%$ rise in T2DM risk factors [8].

TABLE I: MENDELIAN RANDOMIZATION ESTIMATES OF CHILDHOOD OBESITY [8]

\begin{tabular}{ccc}
\hline Factors & No. of Participants & MR-Value (Mean) \\
\hline BMI $\left(\mathrm{Kg} / \mathrm{m}^{2}\right)$ & 322,102 & 0.007 \\
HC $(\mathrm{cm})$ & 212,997 & 0.020 \\
WC $(\mathrm{cm})$ & 232,059 & 0.027 \\
WHR $(\mathrm{cm})$ & 212,159 & 0.132 \\
HDL-c $(\mathrm{mg} / \mathrm{dL})$ & 187,071 & 0.352 \\
LDL-c $(\mathrm{mg} / \mathrm{dL})$ & 172,999 & 0.130 \\
TC $(\mathrm{mg} / \mathrm{dL})$ & 187,238 & 0.456 \\
TG $(\mathrm{mg} / \mathrm{dL})$ & 177,769 & 0.875 \\
\hline
\end{tabular}

The study talks about links of childhood and adulthood BMI showing an inherent vulnerability. The authors believed that this relationship needs to be better discussed through more researches in the recent future to establish the findings. Authors also believed that the genetic factors in the MR Analysis hold no links to $\mathrm{CO}$ and hence it is not a strong instrument in this study. Authors strongly suggested that this research should have more focus on factors like Glucose, LDL-c. Hb, etc. [8]. Also, authors believed that this study in the mere future should be conducted on different ethnic groups and data from each group should be 
compared to better understand the prevalence of T2DM due to $\mathrm{CO}[8]$.

Second study selected for this review, found that out of total number of participants, 45,228 were found to be overweight and 61,358 were obese, which was around $12 \%$ and $16 \%$ respectively [9]. Total 654 cases of T2DM were observed and there was an increase in the number of cases and the mean timeframe between BMI and cases of T2DM was 0.4 [9]. In the year 1994, the T2D occurrence 6.4 which increased to $33.2 \%$ in 2013. The increase in T2DM occurrence was equally observed in both males and females as shown in Table II.

TABLE II: OCCURRENCE RATE (OR) OF T2DM IN CHILDREN FROM UK

\begin{tabular}{ccccccc}
\multicolumn{7}{c}{ DURING 1994-2013 [9] } \\
\hline \multirow{2}{*}{ Factor } & Cases & $\begin{array}{c}\text { OR } \\
(\%)\end{array}$ & Cases & $\begin{array}{c}\text { OR } \\
(\%)\end{array}$ & Cases & $\begin{array}{c}\text { OR } \\
(\%)\end{array}$ \\
\cline { 2 - 7 } & & & & $1999-2003$ & $2004-2008$ \\
\hline Total & 14 & 6.4 & 15.6 & 23.9 & 16.4 & 16.5 \\
Female & 7 & 6.3 & 89 & 27.1 & 90 & 18.3 \\
Male & 7 & 6.4 & 67 & 20.6 & 74 & 14.7 \\
Below 15 & 11 & 5.4 & 89 & 16 & 86 & 11.6 \\
years of age & & & & & & \\
16-25 years & 3 & 19.9 & 67 & 69.2 & 78 & 31 \\
of age & 12 & 7.6 & 99 & 21.3 & 55 & 7.9 \\
Normal BMI & 12.69 .3 \\
Overweight & 0 & 0 & 12 & 15.3 & 22 & 18.2 \\
Obese & 2 & 5.7 & 45 & 40.9 & 87 & 50.7 \\
\hline
\end{tabular}

The Diabetes risk factors was analyzed using Poisson Regression Model. The Incidence Rate Ratios (IRR) for T2D for 2004-2008 and 2009-2013 was 1.7 and 3.3 times higher as rates between 1994-1998 [9]. The study found that occurrence of T2DM was reported in children of age group 5-15 years of age as shown in Table III. It can also be summarized from this study that cases of T2DM increased in obese children during period of 1994-2013 and no significant relation was found between obese children and cases of Type 1 Diabetes Mellitus.

TABLE III: INCIDENCE RATE RATIO (IRR) OF T2DM IN CHILDREN FROM

\begin{tabular}{ccc} 
& UK DURING 1994-2013 [9] & \\
\hline Factor & Unadjusted ORR $(\%)$ & Controlled ORR (\%) \\
\hline Male & 1.5 & 1.5 \\
Female & Not Available & Not Available \\
6-10 years of age & 1.2 & 1.1 \\
11-15 years of age & 2.8 & 2.3 \\
16-25 years of age & 4.8 & 4.7 \\
BMI=Normal & 1.2 & 1.2 \\
Overweight & Not Available & Not Available \\
Obese & 4.5 & 4.3 \\
\hline
\end{tabular}

In controlled settings, obese children accounted for about $47.1 \%$ cases of T2DM and had four times chances of having adulthood diabetes as compared to children with normal BMI as shown in Table IV. This study was conducted on a large group of individuals using the primary electronic health records [9]. The study observed that T2DM occurrence was found to be rising in obese children from the year 1994 to 2013 and on contrary to this, no link has been found between the Type 1 Diabetes Mellitus and children of obese category. T2DM has been found to be associated with intense health burden on the UK society and healthcare services. According to the mentioned study, 1 in every 11 obese children encountered T2DM which accounted for around 415 million children across the country [9].
TABLE IV: BMI RATES IN CHILDREN FROM UK DURING 1994-2013 [9]

\begin{tabular}{cccc}
\hline Factor & Cases & $\begin{array}{c}\text { Unadjusted } \\
\text { Model }\end{array}$ & $\begin{array}{c}\text { Controlled } \\
\text { Model }\end{array}$ \\
\hline & & OR $(\%)$ & OR $(\%)$ \\
Normal BMI & 285 & Not Available & Not Available \\
Overweight & 61 & 1.3 & 1.3 \\
Obese & 308 & 3.9 & 3.8 \\
\hline
\end{tabular}

A study in UK, conducted between 2004-2005 observed that a total of 67 cases of T2DM out of 363 individuals who were below 17 years of age. Another CPRD data showed that children 0-18 years of age show eight times increase in epidemiology of T2DM. This study conducted in Finland, also showed positive associations between the childhood BMI and T2DM [9]. In controlled settings, it was observed that the children and the youth with T2DM were obese and had four times higher than the normal BMI value.

Authors also disclosed several limitations to the above discussed study. The information on BMI, Weight and Height were not presented for all subjects in the CPRD. It is suggested that height and weight collection methods need to be improved, so data for all individuals can be recorded and compared to have better prospective results. Authors did also like to reflect on the major loopholes in this study, that only one aspect of BMI was used to collect data, which could have led to artificial increase in cases of T2DM and the BMI data presented in CPRD did not suffice over time causing disruptions in the probability of the data. Authors also believed that a strict criterion had to be applied on the CPRD cohorts to find about the incidence rates of T2DM among children and youth. Authors also strongly believed that this research study could easily have considered the usage of antibodies of T2DM for the tests and collection of data. Also, this study can only be applied on the individuals in the European region because the data is from the CPRD of UK, which might not be equally applied at global level.

Third study selected for this review mentioned the results around $14 \%$ of men and $9.1 \%$ of women were diagnosed with T2DM [10]. It is shown in the study that the individuals having BMI above mean level at the age of 11 years had the greatest number of cases of T2D which stands at $15.7 \%$ in men and $10.3 \%$ women [10]. These results have been carefully represented in Table V.

According to this study's findings, in individuals of 11 years having BMI levels at birth. BMI, Waist-size, body fat levels were found to be more than usual in individuals with T2DM and in addition to that all these three factors were found to be more in men as compared to women [10]. This study has well represented the positive links between the BMI in children and T2D in 13,345 children who live in Helsinki during 1934-1944. In these children, a sudden rise is seen after 2 years of age due to deposition of fat leading to IR. The mean connotation between BMI and the epidemiology of T2DM was $11.7 \%$ in this age group and was similar to the findings from Finland [10].

In this study, there has been no exact resources to confirm low level of fat mass in individuals during childhood which is increased during adolescent stage and hence causes increase in BMI. The study also reported that there was a sudden increase in BMI among women during childhood and during infancy. But authors believe that further research needs to be conducted to give some strong evidence in its 
regards. According to my viewpoint this study hypothesized about the links of genetic factors to T2DM, but still at the end of the research there remains quite unclear and future research need to be conducted to find the answer to this association [10].

\begin{tabular}{|c|c|c|c|c|}
\hline \multirow[b]{2}{*}{ Body Size } & \multicolumn{2}{|c|}{ BOYS } & \multicolumn{2}{|c|}{ GIRLS } \\
\hline & $\begin{array}{l}\text { BMI above } \\
\text { mean value }\end{array}$ & $\begin{array}{l}\text { BMI below } \\
\text { mean value }\end{array}$ & $\begin{array}{l}\text { BMI above } \\
\text { mean value }\end{array}$ & $\begin{array}{l}\text { BMI above } \\
\text { mean value }\end{array}$ \\
\hline \multicolumn{5}{|c|}{ At Birth } \\
\hline $\begin{array}{l}\text { Length } \\
(\mathrm{cm})\end{array}$ & 50.8 & 50.4 & 50.1 & 49.8 \\
\hline Weight (g) & 35.62 & 33.93 & 34.09 & 32.81 \\
\hline $\begin{array}{c}\text { BMI } \\
\left(\mathrm{kg} / \mathrm{m}^{2}\right)\end{array}$ & 13.80 & 13.30 & 13.60 & 13.20 \\
\hline \multicolumn{5}{|c|}{2 years of age } \\
\hline $\begin{array}{l}\text { Length } \\
(\mathrm{cm})\end{array}$ & 87.00 & 86.20 & 85.80 & 85.10 \\
\hline Weight (g) & 12.80 & 12.00 & 12.30 & 11.50 \\
\hline $\begin{array}{c}\text { BMI } \\
\left(\mathrm{kg} / \mathrm{m}^{2}\right)\end{array}$ & 17.10 & 16.20 & 16.90 & 16.00 \\
\hline \multicolumn{5}{|c|}{11 years of age } \\
\hline $\begin{array}{l}\text { Length } \\
(\mathrm{cm})\end{array}$ & 142.40 & 140.2 & 142.90 & 139.90 \\
\hline Weight (g) & 36.5 & 30.8 & 38.0 & 30.5 \\
\hline $\begin{array}{c}\text { BMI } \\
\left(\mathrm{kg} / \mathrm{m}^{2}\right)\end{array}$ & 18.0 & 15.6 & 18.6 & 15.5 \\
\hline
\end{tabular}

\section{SEARCH STUDY FOR DIABETES IN YOUTH}

SEARCH Study for Diabetes in Youth Study Group has been a successful research study conducted in the year 2014 . This project began in 2000 and funded by Centers for Disease Control and Prevention (CDC) and National Institute of Diabetes and Digestive and Kidney Disease (NIDDK) and talked about gaps in relation to childhood Obesity [11]. This study was conducted in different age groups, sex and ethnicities and it was found that the prevalence of T2DM has been increased in most of these categories. A cohort study to understand the prevalence of T2DM in children was also done. This study had two phases - phase I (conducted between 2000-2005) and phase II (conducted between 2005-2010) and children of 20 years and below were considered [11]. Phase III of this study started in 2010 and was going on at the time of retrieval of this information. This research consisted of registry-based data and a complete cohort study. By 2009, there were an approximately of 3.5 million children under watch and the data is alike to the US youth population register prepared according to ethnicity, age, income, and other socioeconomic failures [12].

The study also found that number of diabetes positive children in 2009 increased to 192,000 and the epidemiology of T2DM in children increased to around $30.5 \%$ for both men and women. It has found that around 5.089 new significant rise in cases were observed for age group of 0.4 years [11]. Around 50\% youth consisting of $30 \%$ children had to be hospitalized due to T2DM manifestations. To report, it was found that T2DM effected children of AfricanAmerican and American Indian ethnicity were from lowincome families [11].

\section{ROLE OF DIET AND PANCREAS INHERENT FACTORS}

Managing diet intake in children has been proven to be fruitful in preventing the prevalence of T2DM in youth of today. For T2DM, higher amounts of calories, fat and carbohydrates have negative effects which tend to increase furthermore due to intake of starches, higher glucose load and added sugars in diet [13]. Randomized Clinical Trials (RCT) have shown that macronutrients-based diet composition plans can be helpful in proving effective for reducing calorie intake and glucotoxicity and lipo-toxicity due to IR lead to Reactive Oxygen Stress (ROS) and proved to be impractical in practice without restricting the calorie intakes and reducing the levels of carbohydrate in the body result in very low levels of HbA1c (Glycosylated Hemoglobin) [13]. It results in low glycemic index of body, high protein levels and increasing levels of Branched-Chain Amino Acids (BCAA) which leads to developing of T2DM [14]. The BCAA in body lead to increased IR and it has been noted that Renal Tubular ability for the reabsorption of glucose is highly increased in individuals with T2DM [15]. And they have around $70 \%$ secretion individuals of insulin due to which there is poor Incretin effect. This effect contributes to T2DM and individuals have poor levels of Incretin and also have enhanced Insulin sensitivity which suppresses working of Glucagon-Dependent Insulinotropic Peptide (GIP) [1].

In children during early lifetime there is lower volume of fat and parenchymal cells in pancreas and remains up to 20 years of age. Individuals who are obese have large size of pancreas and have IR because Obesity and IR a negative association. In individuals with T2DM there is a loss of $\beta$ cells in large numbers which can be identified using Magnetic Resonance Imaging (MRI) [16]. This shows that there is a 33\% lower volume of pancreas of T2DM affected individuals. There is also an increased deposition of fat and Insulin secretion leading to Hyperglycemia [1].

\section{CONCLUSION}

The pathophysiology of Type 2 Diabetes in children with Obesity has been predicted to occur at a higher rate in the coming years and lead to adverse effects on child healthcare. Findings from various scientific studies have helped to enhance the response that the global society is making towards this association in children, but the growing burden has directed the attention of global healthcare organizations towards the need to conduct high-level studies to better understand the early onset of these diseases and the underlying complications to provide a long-term effective approach to help in prevention and management of this group of disease to ensure "Global Health for All".

\section{FUTURE PROSPECTS}

The authors of this review report strongly believe that the gaps in understanding of T2DM, having positive links to Obesity among children and youth of today remain a great challenge to the scientific community and is important as it poses a great healthcare burden on all ethnicities and children of the world falling in the obese category. It is 
therefore recommended that the future studies need to be focused on to address a few important questions that are persistently unanswered:

How and will the T2DM occurrence continue to affect the youth off the world?

Will the T2DM prevalence be seen in all different populations of the world or only in regions with low- and middle- income background?

How can the United Nations Sustainable Development Goal 3 (SDG 3) of "Good Health for All" be achieved if the youth of today is affected by Type 2 Diabetes and Obesity's burden?

Will the world face an epidemic situation due to T2DM prevalence in the society and be able to overcome it?

Can T2DM prevalence and risk factors cause mortality among children who are obese?

It is also believed that the potential answers to the abovementioned questions will set a direction towards the potential solution for the health problems associated with the prevalence and epidemiology of T2DM and CO, especially to safeguard the future generations.

\section{REFERENCES}

[1] Valaiyapathi, B., Gower, B., \& Ashraf, A. P. (2020). Pathophysiology of Type 2 Diabetes in Children and Adolescents Current Diabetes Reviews, $16(3)$. https://doi.org/10.2174/1573399814666180608074510.

[2] Kim, S. Y., England, J. L., Sharma, J. A., \& Njoroge, T. (2011). Impact of Maternal Obesity and Diabetes on Long-Term Health of the Offspring Journal of Diabetes Research 2011, 9. https://doi.org/https://doi.org/10.1155/2011/541308.

[3] Galuska, D. A., Gunn, J. P., O’Connor, A. E., \& Petersen, R. (2018). Addressing Childhood Obesity for Type 2 Diabetes Prevention: Challenges and Opportunities. Diabetes Spectrum, 31(4), 330-335. https://doi.org/https://doi.org/10.2337/ds18-0017.

[4] KayeWooton, A., \& M.Melchior, L. (2017). Obesity and Type 2 Diabetes in Our Youth: A Recipe for Cardiovascular Disease. The Journal Of Nurse Practitioners, 13(3), 222-227 https://doi.org/https://doi.org/10.1016/j.nurpra.2016.08.035.

[5] Zeng, C.-P., Lin, X., Peng, C., Zhou, L., You, H.-M., Shen, J., \& Deng, H.-W. (2019). Identification of novel genetic variants for type 2 diabetes, childhood obesity, and their pleiotropic loci Journal of Human Genetics, 64, 369-377. https://www.nature.com/articles/s10038-019-0577-5.

[6] Davies, N. (2018). Type 2 Diabetes and Obesity: The Link Diabetes Self-Management. https://www.diabetesselfmanagement.com/aboutdiabetes/types-of-diabetes/type-2-diabetes-and-obesity-the-link/.

[7] Silink, M. (2010). The challenge of childhood obesity and diabetes The Lancet, 375(9733). https://doi.org/https://doi.org/10.1016/S01406736(10)61015-X.

[8] Fang, X., Zuo, J., Zhou, J., Cai, J., Chen, C., Xiang, E., Li, H., Cheng, X., \& Chen, P. (2019). Childhood obesity leads to adult type 2 diabetes and coronary artery diseases: A 2-sample mendelian randomization study. 98(32). https://doi.org/10.1097/MD.0000000000016825.

[9] Abbasi, A., Juszczyk, D., van Jaarsveld, C.H., \& Gulliford, M.C. (2017). Body Mass Index and Incident Type 1 and Type 2 Diabetes in Children and Young Adults: A Retrospective Cohort Study Journal of the Endocrine Society, 1(5), 524-537 https://doi.org/https://doi.org/10.1210/js.2017-00044.

[10] Eriksson, J. G., Kajantie, E., Lampl, M., \& Osmond, C. (2015), Trajectories of body mass index amongst children who develop type 2 diabetes as adults Journal of Internal Medicine. https://doi.org/https://doi.org/10.1111/joim.12354.

[11] Jensen, E. T., \& Dabelea, D. (2018). Type 2 Diabetes in Youth: New Lessons from the SEARCH Study. Curr Diab Rep, 18(6). https://doi.org/10.1007/s11892-018-0997-1.

[12] Hamman, R. F., Bell, R. A., Dabelea, D., Jr., R. B. D. A., Dolan, L., Imperatore, G., Lawrence, J. M., Linder, B., Marcovina, S. M., Mayer-Davis, E. J., Pihoker, C., Rodriguez, B. L., \& Saydah, S. (2014). The SEARCH for Diabetes in Youth Study: Rationale,
Findings, and Future Directions Diabetes Care 37(12), 3336-3344 https://doi.org/https://doi.org/10.2337/dc14-0574.

[13] Goss, A. M., Goree, L. L., Ellis, A. C., Chandler-Laney, P. C., Casazza, K., Lockhart, M. E., \& Gower, B. A. (2012). Effects of diet macronutrient composition on body composition and fat distribution during weight maintenance and weight loss The Obesity Society, 21(6), 1139-1142. https://doi.org/https://doi.org/10.1002/oby.20191.

[14] Bloomgarden, Z. (2018). Diabetes and branched-chain amino acids: What is the link? J Diabetes 10(5), 350-352 https://doi.org/10.1111/1753-0407.12645.

[15] Tricò, D., Prinsen, H., Giannini, C., Graaf, R. d., Juchem, C., Li, F. Caprio, S., Santoro, N., \& Herzog, R. I. (2017). Elevated $\alpha$ Hydroxybutyrate and Branched-Chain Amino Acid Levels Predict Deterioration of Glycemic Control in Adolescents The Journal of Clinical Endocrinology \& Metabolism, 102(7), 2473-2481. https://doi.org/https://doi.org/10.1210/jc.2017-00475.

[16] Michaliszyn, S. F., Mari, A., Lee, S., Bacha, F., Tfayli, H., Farchoukh, L., Ferrannini, E., \& Arslanian, S. (2014). $\beta$-Cell Function, Incretin Effect, and Incretin Hormones in Obese Youth Along the Span of Glucose Tolerance from Normal to Prediabetes to Type 2 Diabetes Diabetes Care, 63(11), 3846-3855 https://doi.org/https://doi.org/10.2337/db13-1951. 